\title{
Análisis de la actividad laboral de los estu- diantes universitarios y de sus efectos sobre el rendimiento académico
}

\section{Hipólito Simón ${ }^{1}$, José Manuel Casado Díaz ${ }^{1}$, Juan Luis Castejón Costa ${ }^{2}$}

${ }^{1}$ Departamento de Análisis Económico Aplicado e Instituto Interuniversitario de Economía Internacional, Universidad de Alicante, Alicante ${ }^{2}$ Departamento de Psicología Evolutiva y Didáctica, Universidad de Alicante, Alicante

\section{España}

Correspondencia: Hipólito Simón Pérez. Departamento de Análisis Económico Aplicado. Aulario I. Universidad de Alicante. Carretera San Vicente del Raspeig s/n. 03690. San Vicente del Raspeig - Alicante. España. E-mail: hsimon@ua.es

(C) Education \& Psychology I+D+i and Ilustre Colegio Oficial de la Psicología de Andalucía Oriental (Spain) 


\section{Resumen}

Introducción. La investigación tiene dos objetivos: caracterizar a los estudiantes universitarios que simultanean trabajo y estudios y el tipo de trabajo que desempeñan, por un lado, y analizar el efecto que tiene trabajar sobre distintas medidas de su rendimiento académico, por otro.

Método. El análisis se desarrolla a partir de información individual procedente de una encuesta $(\mathrm{N}=464)$ combinada con registros universitarios. Se han estimado modelos en los que se consideran distintas medidas del desempeño académico y en los que se controla por un amplio conjunto de factores (incluyendo características socioeconómicas así como medidas de motivación y de esfuerzo académico), distinguiendo entre distintos niveles de intensidad de la actividad laboral. Las técnicas usadas han sido la regresión logística, mínimos cuadrados ordinarios y variables instrumentales.

Resultados. Se confirma, en primer lugar, que una proporción significativa de los estudiantes universitarios españoles simultanea los estudios con un trabajo remunerado, siendo muy frecuente que trabajen de forma habitual durante periodos prolongados. Se observa, además, que es frecuente que quienes trabajan lo hagan motivados por necesidad y en tareas no relacionadas con el contenido de sus estudios, y que la propensión a trabajar es mayor entre los estudiantes de mayor edad, los extranjeros y los de mayor motivación. En segundo lugar, los resultados obtenidos sugieren que, en contraste con la propia percepción manifestada por los estudiantes, trabajar regularmente no tiene aparentemente un impacto significativo en sus resultados académicos.

Conclusión. El estudio añade evidencia sobre un tema relevante pero muy escasamente analizado en el caso español, sobre el que ni la teoría ni los estudios empíricos previos sobre casos internacionales ofrecen resultados concluyentes. Los resultados son útiles para la consideración que el desempeño de una actividad laboral debe recibir en el diseño de los itinerarios seguidos por los estudiantes universitarios.

Palabras Clave: Rendimiento académico, resultados académicos, educación superior, empleo estudiantil, motivación estudiantil, España. 


\section{Abstract}

Introduction. The research has two objectives: to characterise college students who combine work and studies, and their jobs, on one hand; and to analyse the effect of work on various measures of academic performance, on the other.

Method. The study is developed using individual information derived from a survey $(\mathrm{N}=464)$ and from university records. We have estimated models that consider diverse measures of academic performance and control for a wide set of factors (including socio-economic characteristics and diverse measures of motivation and academic effort), distinguishing between different levels of intensity in the labour activity. The techniques used are logistic regression, ordinary least squares and instrumental variables.

Results. The evidence obtained confirms, first, that a noticeable proportion of Spanish university students have a paid job, and often work regularly for extended periods. It also is common for those who work to do so motivated by necessity, and to perform tasks unrelated to the content of their studies. Moreover, the likelihood of working is higher among older students, foreigners and those who have higher levels of motivation. Secondly, the results suggest that, in contrast to the perceptions expressed by students, working regularly does not appear to have a significant impact on their academic performance.

Conclusion. The article provides additional evidence on a very relevant issue that has however received very little attention in the Spanish case and for which neither theoretical studies nor previous empirical research have reached conclusive results. The article provides useful support for the consideration of employment-related issues in the designing of the itineraries followed by university students.

Keywords. Academic achievement, academic performance, academic outcomes, higher education, student employment, student motivation, Spain. 


\section{Introducción}

Si bien la incidencia del fenómeno varía significativamente entre países, es muy habitual que los estudiantes universitarios estudien a la vez que trabajan. Aunque es cierto que en el caso español la proporción de estudiantes que compatibilizan estudio y trabajo es menor que en otros países avanzados, se trata de una situación que afecta a un porcentaje significativo de los estudiantes de nuestro país, ya que en torno un tercio de quienes cursan estudios superiores en España también trabaja (Quintini, 2015).

Este fenómeno ha sido analizado en profundidad para otros países como Estados Unidos o el Reino Unido (véanse por ejemplo Richardson, Kemp, Malinen \& Haultain, 2013; Curtis, 2007, o Nonis \& Hudson, 2006). En líneas generales, la evidencia al respecto del posible efecto de trabajar sobre el rendimiento académico de los estudiantes universitarios no es concluyente, ya que existen estudios que sugieren tanto una influencia positiva como una negativa (Riggert, Boyle, Petrosko, Ash, \& Rude-Parkins, C., 2006, y Stern \& Nakata, 1991). Esta circunstancia contrasta con el carácter más concluyente de la evidencia sobre el efecto positivo que trabajar durante los estudios universitarios tiene sobre la carrera laboral y los salarios futuros, así como sobre el efecto negativo de la actividad laboral sobre la permanencia en la universidad o la duración de los estudios (puede encontrarse una revisión exhaustiva de la literatura relacionada con estas cuestiones en Riggert et al., 2006). En el caso concreto de España, donde esta cuestión ha sido muy poco analizada, los resultados de Ruesga, Da Silva \& Monsueto (2014) sugieren que trabajar afecta negativamente al desempeño académico de los estudiantes, pero únicamente para quienes trabajan 15 o más horas semanales.

El objetivo de este artículo es doble. En primer lugar se desarrolla una caracterización de los estudiantes universitarios españoles que también trabajan y del tipo de trabajo que desempeñan. En segundo lugar se analiza el efecto de la actividad laboral sobre el rendimiento académico de dichos estudiantes. Dada la escasez de precedentes de este tipo de estudios para el caso español, entendemos que los resultados del estudio podrían permitirán completar esta laguna de conocimientos para nuestro país y, entre otras cuestiones, ayudar a determinar la pertinencia de la necesidad de diseñar sistemas de aprendizaje y evaluación que garanticen la igualdad de oportunidades y formación así como de éxito en los resultados de aprendizaje para todos los perfiles de estudiantes, incluyendo también a quienes trabajan. El análisis empírico se desarrolla a partir de una base de datos con información muy variada sobre las carac- 
terísticas socioeconómicas, familiares y académicas, así como sobre los empleos de los estudiantes. Dicha base de datos ha sido creada combinando datos individuales procedentes de una encuesta con otros obtenidos de registros universitarios.

Son varias las aportaciones de la investigación. En primer lugar, la caracterización detallada del fenómeno de simultanear trabajo y estudios universitarios aporta información novedosa sobre una cuestión para la que, en contraste con lo que sucede en otros países, apenas existe evidencia para el caso español, a pesar de afectar a una proporción significativa de los estudiantes. En segundo lugar, se ofrece evidencia que complementa la obtenida en el único estudio previo que ha examinado el impacto de la actividad laboral sobre el rendimiento académico de los estudiantes universitarios españoles (Ruesga et al., 2014). En relación con dicho trabajo, este análisis explora distintas medidas alternativas del desempeño académico e incluye un amplio conjunto de variables de control que incluyen otros factores que, de acuerdo con la literatura relevante, pueden influir en el rendimiento académico, tales como la motivación de los estudiantes o su esfuerzo académico.

\section{La relación entre trabajo y estudio universitario}

La evidencia existente actualmente sobre en qué medida los estudiantes universitarios estudian a la vez que trabajan corresponde en buena medida a un número reducido de países, ya que la evidencia comparativa internacional es muy escasa. La principal excepción es Quintini (2015) quien, a partir de la evidencia comparable entre economías procedente de la Survey of Adult Skills (PIAAC), documenta el fenómeno en 23 países o regiones avanzadas, mostrando que en torno al 60\%-70\% de los estudiantes en los países anglosajones y los países nórdicos y entre el 20\% y el 30\% de los del Sur de Europa trabaja. A pesar de la significativa extensión del fenómeno en muchos países, y de la relevancia del mismo para estudiantes, instituciones educativas y el conjunto de la sociedad, ciertas cuestiones relevantes, como la relación entre el desempeño de un trabajo y el rendimiento académico de los estudiantes universitarios han sido poco investigadas, con un número de estudios relativamente modesto de los cuales la inmensa mayoría tiene un carácter empírico. En consecuencia, aunque la literatura actual responde a algunas importantes cuestiones sobre los efectos del empleo de los estudiantes universitarios, existen importantes interrogantes abiertos (puede encontrarse una revisión exhaustiva reciente de los estudios sobre esta cuestión Riggert et al., 2006; para una revisión anterior de la literatura, véase Lyons, Krachenberg \& Henke, 1986). 
Desde una perspectiva teórica existen argumentos que defienden la existencia tanto de un hipotético efecto positivo del desempeño de la actividad laboral sobre el rendimiento académico como de un efecto en sentido contrario. Así, los efectos negativos de trabajar pueden derivarse de un modelo de suma cero, donde el tiempo dedicado a trabajar va en detrimento del dedicado a estudiar, mientras que, por el contrario, los efectos positivos se pueden argumentar a partir de un modelo de orientación primaria, basado en la expectativa de que los estudiantes más motivados y con más habilidades pueden ser precisamente los más capaces de equilibrar el trabajo remunerado y las responsabilidades académicas (Warren, 2002).

Tampoco la evidencia empírica que proporcionan los estudios existentes al respecto del posible efecto de trabajar sobre los logros académicos de los estudiantes universitarios es concluyente $^{1}$. Así, mientras que algunos estudios sugieren que trabajar tiene un impacto perjudicial sobre el rendimiento (véanse, por ejemplo, Tyler, 2003; Stinebricker \& Stinebricker, 2003, o Hawkings et al., 1995), otros apuntan a que el empleo tiene un impacto neutral o incluso beneficioso (Furr \& Elling, 2000; Nonis y Hudson, 2006; Pascarella, Bohr, Amaury, Desler \& Zusman, 1994, y Pascarella, Edison, Nora, Hagedorn \& Terenzini, 1998; Canabal; 1998; Hammes \& Haller, 1983, y Gleason, 1993), si bien es posible que un impacto negativo sea más evidente en el caso de jornadas laborales más extensas. En cualquier caso, cabe destacar que los efectos identificados son en todos los casos de pequeña magnitud. Estas diferencias en el sentido de los resultados pueden deberse a diferencias metodológicas entre las investigaciones, pero también a diferencias entre los distintos contextos analizados, lo que subraya la relevancia de extender estos análisis a otros países (Riggert et al., 2006).

En otro orden de cosas, cabe destacar que la forma de medir la actividad laboral del alumnado universitario presenta diferencias significativas entre estudios (para más detalles véase Riggert et al., 2006). Así, mientras que existen trabajos que consideran el conjunto de estudiantes que trabajan, sin atender a las características del empleo, otros diferencian entre trabajos a jornada parcial y completa en función del número de horas trabajadas, utilizando para ello distintos umbrales, siendo uno de los más habituales el de 30 horas de jornada labo-

\footnotetext{
${ }^{1}$ Esta circunstancia contrasta, como se ha señalado, con la evidencia más concluyente existente sobre el efecto positivo del empleo estudiantil sobre los logros profesionales posteriores, ya que ciertos estudios sugieren que trabajar un número moderado de horas puede ayudar a los resultados laborales al finalizar los estudios (Dundes \& Marx, 2006), así como en el sentido de que la experiencia laboral adquirida mientras se estudia beneficia el 
ral semanal (véanse, por ejemplo, Pike, Kuh \& Massa-McKinley, 2008; Lillydahl, 1990, o Hood, Craig \& Ferguson, 1992). En el mismo sentido, algunos análisis distinguen entre las distintas motivaciones que llevan a trabajar (Wenz y Yu, 2010); el grado de relación entre el tipo de trabajo y los estudios cursados (Stern, Finkelstein, Urquiola \& Cagampang, 1997) o la propia percepción de los estudiantes sobre el impacto de trabajar en sus resultados académicos (Curtis, 2007). Por su parte, en lo que respecta a la medición de los logros académicos, cabe destacar que, si bien una parte de los estudios que ha examinado la relación entre empleo y rendimiento ha utilizado medidas alternativas, como el tiempo requerido para completar los estudios o la tasa de abandono (véanse, por ejemplo, Canabal, 1998, o Gleason, 1993), una parte significativa de los mismos lo ha hecho utilizando como variable dependiente una medida de los resultados académicos promedio del estudiante en su titulación (siendo la más habitual en los estudios para Estados Unidos el GPA, o grade point average).

En el caso de España, la evidencia sobre el fenómeno de los estudiantes universitarios que trabajan es muy escasa. Así, si bien existen numerosas investigaciones sobre los determinantes del desempeño académico y del fracaso escolar de los estudiantes universitarios españoles (véase, por ejemplo, la revisión de estos estudios en Tejedor y García-Valcárcel, 2007), los análisis sobre el efecto de desempeñar un trabajo remunerado son marcadamente escasos y, hasta donde conocemos, se limitan al estudio de Ruesga et al. (2014). Estos autores basan su análisis empírico en una encuesta cumplimentada por estudiantes universitarios de un amplio conjunto de universidades españolas y en el uso de modelos econométricos para estimar los determinantes de la entrada de los estudiantes universitarios en el mercado de trabajo y sus implicaciones sobre el desempeño académico. En el primer caso, mediante la estimación de un modelo logit multinomial encuentran evidencia de que los principales determinantes de trabajar serían la edad, la experiencia laboral anterior al comienzo de los estudios universitarios y las transferencias monetarias de las familias a los estudiantes. En el segundo, mediante un modelo de variables instrumentales concluyen que trabajar afecta negativamente al desempeño académico de los estudiantes que tienen una jornada laboral superior a 15 horas semanales, si bien sus resultados muestran también que estar empleado puede ser positivo cuando la jornada laboral es inferior a 15 horas o cuando el estudiante tiene experiencia laboral previa a la entrada en la universidad.

desempeño laboral futuro, especialmente en el caso de que el trabajo esté relacionado con el campo de estudio (Beffy, Fougère \& Maurel, 2009). 


\section{Método}

\section{Participantes}

La población objeto de estudio en la investigación está constituida por el alumnado matriculado en la asignatura Economía Mundial del grado en Administración y Dirección de Empresas (ADE) de la Universidad de Alicante y del doble grado Turismo-ADE (TADE) de dicha universidad. Se trata de una asignatura obligatoria de segundo curso (primer semestre) en la que se matricularon 521 estudiantes para ambos grados en el curso 2015/2016.

\section{Instrumentos}

En relación con las variables utilizadas en el análisis, se han empleado tres medidas distintas del desempeño académico que incluyen tanto medidas de los resultados académicos globales (nota media en la titulación, medida es una escala de 0 a 10, y número de créditos aprobados por año matriculado) como de los resultados académicos obtenidos específicamente durante el semestre de realización de la encuesta (número de créditos aprobados en el semestre $)^{2}$.

El resto de variables utilizadas en el análisis empírico engloban atributos socioeconómicos (sexo; edad, nacionalidad -distinguiendo entre española, extranjera y doble nacionalidad-; posición en el hogar -diferenciando entre hijo y otra-; nivel de estudios de ambos progenitores -estudios terciarios u otro tipo de estudios- y nivel relativo de renta -autoclasificación en cuartiles de la distribución de renta del conjunto de España- ${ }^{3}$ ); académicos (si se es repetidor; si se cursa el grado de forma vocacional; el grado de asistencia a clase y el número de horas de preparación académica por semana) y variables laborales. Estas últimas incluyen la relación con el empleo (trabaja habitualmente/trabaja ocasionalmente/no trabaja); el número de horas trabajadas por quienes lo hacen habitualmente; la percepción sobre la relación con el empleo durante el curso académico; el tipo de ocupación (cualificada/semicualificada/no cualificada); el sector de actividad del empleo; los motivos para trabajar (incluyendo motivos como la necesidad o ayudar a la economía familiar) y variables relativas a si se trabaja habitualmente en situación legal; si se podría cursar estudios sin ingresos del empleo; si el empleo

\footnotetext{
${ }^{2}$ Alternativamente, se han considerado otras medidas de rendimiento, como el número de créditos totales aprobados en el grado o la calificación y la probabilidad de aprobar la asignatura de Economía mundial, respectivamente, pero los resultados del análisis son esencialmente similares. Los mismos están disponibles por parte de los autores ante su requerimiento.

${ }^{3}$ La misma se basa en el resultado arrojado por la aplicación online de la OCDE “¿Cuál es su parte?” (http://bd9da336ae.url-de-test.ws/index_es.php?v=; OCDE, 2015) en la que se introducen los ingresos y el nú- 
está relacionado con el contenido del grado; si afecta negativamente al tiempo para preparar las asignaturas del grado y si el rendimiento académico mejoraría si no trabajara (en todos los casos se trata de variables tipo Likert con valores entre 1 y 5 , donde 1 es total desacuerdo y 5 total acuerdo con la pregunta).

Por último, también se han considerado distintas medidas de la motivación de los estudiantes. Como medida original de dicha motivación se ha empleado una escala reducida de 8 elementos elaborada a partir de dos cuestionarios de motivación de logro: el cuestionario MAE (Motivación y ansiedad de ejecución) de Pelechano (1975) y el cuestionario MAPE-II de Montero y Alonso (1992). Del cuestionario MAE se tomaron los cuatro ítems con más saturación en el factor Tendencia a sobrecarga de trabajo (los cuales también están presentes en el factor Alta capacidad de trabajo y rendimiento del MAPE-II) y cuatro ítems del factor Ambición del MAPE-II (que también están presentes en el MAE). Cabe destacar que estos factores relativos a la motivación de logro han mostrado una relación alta con el rendimiento académico y el desempeño laboral en los análisis empíricos (Alonso, 1992; Castejón, 2014; Pelechano, 1975).

El análisis factorial realizado sobre los ocho elementos, empleando para ello el método de extracción de componentes principales y de rotación varimax, puso de manifiesto la existencia de dos factores. El primer factor, que explica el 36,7\% de la varianza, que se puede definir como ambición (ya que incluye, por ejemplo, ítems como "siempre he tenido pretensiones ambiciosas en el trabajo que he hecho" o "creo que soy bastante ambicioso), con saturaciones factoriales que oscilaron entre 0,85 y 0,67 . El segundo factor explica el $18,8 \%$ de la varianza, con saturaciones factoriales que van de 0,82 a 0,37 , y se define como alta capacidad de trabajo y rendimiento (como evidencian el ítem "frecuentemente tomo demasiado trabajo a la vez" o el ítem 3 "cuando trabajo en colaboración con otros, frecuentemente rindo más que ellos"). La fiabilidad de consistencia interna, estimada mediante el coeficiente alfa de Cronbach fue $\alpha=0,79$ para el factor de ambición y $\alpha=0,69$ para el factor de alta capacidad de trabajo y rendimiento.

mero de miembros del hogar y se obtiene el percentil que ocupa el hogar en la distribución de la renta en España usando datos procedentes de la OECD Income Distribution Database. 
Un análisis factorial de segundo orden realizado sobre las puntuaciones factoriales obtenidas en el análisis factorial de primer orden con el método de componentes principales y rotación oblimin directo puso de manifiesto la existencia de un único factor que explicó el $65,2 \%$ de la varianza. La fiabilidad de consistencia interna de los ocho ítems que componen la escala fue asimismo moderadamente alta $(\alpha=0,74)$. Por tanto, se calculó una puntuación total consistente en la suma de los ocho ítems de la escala, que es la variable de motivación finalmente utilizada en los análisis empíricos.

\section{Procedimiento}

Para la obtención de la información sobre las variables utilizadas en los análisis se combinaron, para cada individuo, los resultados de una encuesta realizada en el primer semestre de dicho curso con información individual procedente de registros de la Universidad de Alicante ${ }^{4}$.

Para el desarrollo de la encuesta se eligió una aproximación de tipo censal. Así, todos los individuos de la población fueron invitados a cumplimentar un cuestionario accesible a través de internet, que obtuvo una tasa de respuesta muy elevada (89,1\%; 464 respuestas). La elaboración de la encuesta se produjo en el seno de una red de docencia financiada por la Universidad de Alicante, de la que forman parte diversos miembros del colectivo de personal docente e investigador con experiencia en la investigación sobre docencia así como dos estudiantes, con el fin de facilitar el intercambio de experiencias donde estén implicadas todas las partes.

\section{Análisis de datos}

\section{Análisis descriptivos}

Las tablas A.1 y A.2 del anexo contienen los descriptivos de las variables utilizadas en la investigación. Del total de estudiantes que componen la muestra (464), el 34,9\% (162) desempeñaba un empleo remunerado, lo que confirma que se trata en general de un fenómeno relevante en nuestro país, ya que afecta a una proporción significativa de los estudiantes uni-

\footnotetext{
${ }^{4}$ El cuestionario utilizado incluía una advertencia de acuerdo con la cual el alumnado que respondió a la encuesta aceptaba que los datos facilitados fueran combinados con otros procedentes de los registros de la Universidad de Alicante con el fin de desarrollar la investigación. Los trámites posteriores incluyeron la apertura de un expediente de cesión de datos de carácter personal por parte de la Universidad de Alicante, la cual facilitó la información solicitada tras el informe favorable emitido por el Servicio Jurídico.
} 
versitarios. El trabajo remunerado tiene un carácter habitual para el 16,2\% de los estudiantes (75) y un carácter ocasional para el 18,7\% (87), estando en torno al 37\% la proporción de los primeros que trabaja a jornada completa (entendida, como es habitual en estudios similares, como trabajar al menos 30 horas).

La tabla A.1 contiene información descriptiva detallada sobre el rendimiento académico y las características socioeconómicas y académicas, así como las relacionadas con la motivación, diferenciando entre los estudiantes que únicamente estudian y aquellos que tienen un trabajo remunerado. Así, en relación con el principal colectivo de interés en la investigación, los estudiantes que trabajan habitualmente, se constata en primer lugar que presentan valores más reducidos en los indicadores de rendimiento académico, siendo las diferencias significativas (al 5\% de significatividad) en dos de los tres indicadores de rendimiento académico (nota media del grado y número de créditos aprobados en el semestre). En el mismo sentido, los estudiantes con trabajo habitual presentan características socioeconómicas diferenciadas en comparación con quienes sólo estudian pues, entre otras circunstancias, tienen una mayor edad (24,8 frente a 20,2 en el caso de los estudiantes que únicamente estudian) y son en mayor medida hombres e individuos con nacionalidad extranjera; tienen padres con niveles de estudio ligeramente inferiores y tienen una mayor presencia relativa en hogares con niveles de renta media-baja). Además, presentan mayores niveles de motivación (la diferencia es significativa al 1\%). Finalmente, en cuanto a las variables académicas, se constata que en términos relativos los estudiantes que trabajan habitualmente están matriculados de menos créditos; presentan una menor asistencia a clase; dedican en general menos horas semanales al trabajo académico y cursan el grado de forma menos vocacional.

La tabla A.2, por su parte, muestra información adicional sobre las características de los estudiantes que trabajan y de sus empleos, diferenciando entre quienes tienen un trabajo habitual (y, dentro de éstos, los que trabajan a jornada completa) y, con fines comparativos, quienes trabajan sólo ocasionalmente. Se constata así que el número promedio de horas de trabajo semanales de los estudiantes que trabajan habitualmente es relativamente elevado, en torno a 22 horas (36,5 horas para quienes lo hacen a jornada completa). Como cabría esperar, quienes tienen un trabajo habitual declaran mayoritariamente que se dedican a trabajar y estudiar o principalmente trabajar, muy especialmente quienes lo hacen a jornada completa (en contraste, la práctica totalidad de quienes trabajan de forma ocasional describe su situación 
como única o principalmente estudiar). Asimismo, tanto quienes trabajan de forma habitual como quienes lo hacen ocasionalmente desempeñan mayoritariamente trabajos en el sector servicios (96 y 88\%, respectivamente) y alrededor del $70 \%$ lo hace en ocupaciones que requieren niveles de cualificación intermedios. Una proporción no desdeñable de quienes trabajan lo hace motivado por necesidad y, especialmente, ayudar a la economía familiar, declarando en torno a la mitad de ellos que están muy o bastante de acuerdo con que no sería posible para ellos estudiar sin los ingresos asociados a su trabajo. A su vez, lo hacen mayoritariamente en situación legal (en torno al 60\% de los mismos declara hacerlo siempre o casi siempre) y en trabajos no relacionados con el contenido de sus estudios (en el 50\% de los casos no hay ninguna o casi ninguna relación). Finalmente, cabe destacar que entre los estudiantes que trabajan de forma habitual existe una percepción generalizada de que trabajar les resta tiempo para su preparación académica (el 60\% declara que le afecta bastante o mucho) y les afecta negativamente en su desempeño académico (el 80\% declara que su rendimiento académico mejoraría bastante o mucho si no trabajara).

\section{Análisis multivariante}

Los análisis multivariantes realizados dan cuenta de los dos objetivos que persigue este trabajo: la caracterización de los estudiantes universitarios españoles que simultanean trabajo y estudios y el análisis del efecto de trabajar sobre el rendimiento académico de dichos estudiantes.

Comenzando por el primero de los objetivos, y con la finalidad de examinar qué atributos están asociados de forma significativa con una mayor propensión de los estudiantes a trabajar habitualmente tras eliminar los efectos de composición, la tabla 1 recoge los resultados obtenidos mediante la estimación de un modelo de regresión logística (los resultados son muy similares utilizando un modelo probit) donde la variable dependiente es una variable dicotómica que refleja si el estudiante trabaja habitualmente (o alternativamente si trabaja a tiempo completo, 30 horas o más) y como variables independientes se consideran aquellas características socioeconómicas o relacionadas con la motivación que podrían influir potencialmente en la decisión de trabajar (pueden encontrarse análisis similares en Richardson et al., 2013, o Quintini, 2015). 
Tabla 1. Determinantes de trabajar habitualmente

\begin{tabular}{|c|c|c|}
\hline & $\begin{array}{c}\text { Trabajas } \\
\text { habitualmente }\end{array}$ & $\begin{array}{c}\text { Trabajas } \\
\text { habitualmente } \\
30 \text { horas o } \\
\text { más }\end{array}$ \\
\hline Hombre & $\begin{array}{l}0.015 \\
(0.033)\end{array}$ & $\begin{array}{l}0.025 \\
(0.021)\end{array}$ \\
\hline Edad & $\begin{array}{l}0.033 \\
(0.005) * * *\end{array}$ & $\begin{array}{l}0.026 \\
(0.003) * * *\end{array}$ \\
\hline Nacionalidad española & $\begin{array}{l}-0.097 \\
(0.045)^{* *}\end{array}$ & $\begin{array}{l}0.026 \\
(0.029)\end{array}$ \\
\hline Padre con estudios terciarios & $\begin{array}{l}-0.001 \\
(0.039)\end{array}$ & $\begin{array}{l}-0.010 \\
(0.025)\end{array}$ \\
\hline Madre con estudios terciarios & $\begin{array}{l}-0.028 \\
(0.039)\end{array}$ & $\begin{array}{l}0.027 \\
(0.025)\end{array}$ \\
\hline Posición en el hogar: hijo & $\begin{array}{l}-0.154 \\
(0.081)^{*}\end{array}$ & $\begin{array}{l}-0.088 \\
(0.051)^{*}\end{array}$ \\
\hline Renta del hogar media-baja & $\begin{array}{l}0.071 \\
(0.051)\end{array}$ & $\begin{array}{l}-0.025 \\
(0.032)\end{array}$ \\
\hline Renta del hogar media-alta & $\begin{array}{l}0.064 \\
(0.051)\end{array}$ & $\begin{array}{l}-0.013 \\
(0.033)\end{array}$ \\
\hline Renta del hogar alta & $\begin{array}{l}0.039 \\
(0.071)\end{array}$ & $\begin{array}{l}0.022 \\
(0.045)\end{array}$ \\
\hline Motivación & $\begin{array}{l}0.008 \\
(0.003) * *\end{array}$ & $\begin{array}{l}0.005 \\
(0.002) * *\end{array}$ \\
\hline Vocación en el grado & $\begin{array}{l}0.009 \\
(0.017)\end{array}$ & $\begin{array}{l}-0.007 \\
(0.011)\end{array}$ \\
\hline Constante & $\begin{array}{l}-0.621 \\
(0.195) * * *\end{array}$ & $\begin{array}{l}-0.542 \\
(0.124) * * *\end{array}$ \\
\hline$R^{2}$ & 0.21 & 0.24 \\
\hline$N$ & 464 & 464 \\
\hline
\end{tabular}

Nota. $* p<0.1 ; * * p<0.05 ; * * * p<0.01$

Los resultados obtenidos revelan que la probabilidad de trabajar habitualmente es significativamente mayor entre los estudiantes de más edad; con nacionalidad extranjera (el coeficiente estimado es significativo en este caso al 5\%) y aquellos que ocupan una posición en el hogar distinta a la de hijo (el coeficiente estimado es significativo al 10\%). Asimismo, se constata que los estudiantes que trabajan presentan en general una mayor motivación (el coeficiente estimado es significativo al 5\%). Por el contrario, no se observa una influencia significativa de otros atributos como el género, el nivel educativo de los padres o el nivel de renta del hogar. Los resultados son, por su parte, muy similares en general para quienes trabajan habitualmente 30 horas o más, con la única diferencia de la nacionalidad, que en este caso no resulta significativa.

Por su parte, se han usado técnicas multivariantes para estimar por separado varios modelos que tienen como finalidad examinar en qué medida trabajar habitualmente (o, alter- 
nativamente, hacerlo a tiempo completo), influye en el rendimiento académico de los estudiantes $^{5}$. En dichas estimaciones se han utilizado como variables dependientes distintas medidas de logro académico, relacionadas o bien con los logros académicos globales (nota media en el grado y número de créditos totales aprobados en relación con el número de años matriculado en el grado) o bien con sus logros durante el semestre en el que se recogieron los datos (créditos aprobados en el semestre). Como variables de control se han considerado características socioeconómicas (edad, nacionalidad, nivel de estudios de los padres, posición en el hogar y nivel de renta del hogar) y ciertas características académicas (si el estudiante es repetidor; diversas medidas del esfuerzo académico y efectos fijos por clase).

Uno de los problemas potenciales en la estimación de este tipo de modelos es la posible endogeneidad de la variable dependiente, ya que si tanto la decisión de trabajar habitualmente como el rendimiento académico resultan influidos por un mismo conjunto de factores inobservados, la estimación de los modelos mediante mínimos cuadrados ordinarios no sería apropiada dado que se incumpliría el requisito de ausencia de correlación entre la variable dependiente y el término de error. Esta circunstancia es posible en este contexto, en tanto en que puede darse la circunstancia de que sean precisamente los estudiantes más motivados y con más capacidad quienes consiguen equilibrar mejor el trabajo remunerado y las responsabilidades académicas (modelo de orientación primaria), lo que ha llevado a distintos autores a estimar el efecto de la actividad laboral sobre el rendimiento académico mediante técnicas de variables instrumentales (véanse, por ejemplo, Stinebricker \& Stinebricker, 2003, o Ruesga et al., 2014 para el caso español). En la medida, no obstante, en que el uso de esta última técnica presenta ciertas carencias, entre las que destaca la dificultad de encontrar instrumentos apropiados (véase al respecto Angrist \& Pischke, 2009) y los problemas asociados a la aplicación de la misma a muestras pequeñas (pues los estimadores de variables instrumentales están innatamente sesgados y sus propiedades en muestras finitas son a menudo problemáticas: Baum, 2006), la estrategia empírica adoptada en este trabajo consiste es realizar las estimaciones de los modelos tanto mediante mínimos cuadrados ordinarios (introduciendo variables de control que permiten aproximar la influencia de la motivación y la capacidad) como mediante variables instrumentales.

\footnotetext{
${ }^{5}$ También se han realizado estimaciones con la finalidad de examinar si el número de horas trabajadas por los estudiantes que trabajan habitualmente influye en su rendimiento académico (pueden encontrarse análisis similares en Lang, 2012, o Richardson et al., 2013). El sentido de los resultados obtenidos no varía. Los mismos están disponibles por parte de los autores ante su requerimiento. 
Así, se ha estimado en primer lugar mediante mínimos cuadrados ordinarios, introduciendo entre las variables explicativas dos medidas de la motivación: la motivación general medida a partir de la puntuación total de las medidas de motivación y una variable que refleja en qué medida se cursa el grado de forma vocacional (tablas 2 y 3). Alternativamente, se han estimado los mismos modelos introduciendo adicionalmente como variables de control de la capacidad de los estudiantes la nota media obtenida en el bachillerato y en la prueba de acceso a la universidad ${ }^{6}$ (tabla 4$)^{7}$. Los resultados de los análisis de regresión muestran que ni trabajar habitualmente (tablas 2 y 4) ni hacerlo con una jornada prolongada (tablas 3 y 4) presenta una relación estadísticamente significativa con ninguna medida de logro académico de los estudiantes. En lo que respecta a las variables de control, las únicas que muestran una relación significativa con el rendimiento académico en todos los modelos son la edad y una de las medidas de capacidad (la nota media del bachillerato). En sentido contrario, no parecen influir en el rendimiento académico ni otras características personales o familiares (incluyendo la educación de los padres, el nivel de renta del hogar o la posición en el hogar), ni las medidas de motivación ni, en general, tampoco las variables académicas.

Tabla 2. Influencia de trabajar habitualmente en el rendimiento académico de los estudiantes

\begin{tabular}{llll}
\hline & $\begin{array}{c}\text { Nota } \\
\text { Media } \\
\text { grado }\end{array}$ & $\begin{array}{c}\text { Créditos } \\
\text { aprobados } \\
\text { por año en grado }\end{array}$ & $\begin{array}{c}\text { Créditos aproba- } \\
\text { dos en semestre }\end{array}$ \\
\hline Trabajo habitual & -0.143 & 0.196 & 0.479 \\
& $(0.099)$ & $(-2.344)$ & $(-1.324)$ \\
Hombre & 0.011 & -5.414 & -0.796 \\
& $(0.060)$ & $(1.573)^{* * *}$ & $(0.889)$ \\
Edad & 0.029 & 0.755 & 0.067 \\
Nacionalidad española & $(0.009) * * *$ & $(0.228)^{* * *}$ & $(0.129)$ \\
Padre con estudios terciarios & 0.130 & 0.809 & 0.475 \\
& $(0.084)$ & $(2.218)$ & $(1.253)$ \\
Madre con estudios terciarios & -0.103 & -0.671 & -0.470 \\
& $(0.072)$ & $(1.903)$ & $(1.075)$ \\
Renta del hogar media-baja & 0.070 & 0.250 & 1.146 \\
& $(0.072)$ & $(1.907)$ & $(1.077)$ \\
Renta del hogar media-alta & 0.016 & -0.480 & -1.392 \\
& $(0.094)$ & $(2.465)$ & $(1.392)$ \\
Renta del hogar alta & -0.085 & -0.314 & -0.293 \\
& $(0.095)$ & $(2.498)$ & $(1.411)$ \\
& 0.026 & 1.394 & 2.930 \\
& $(0.131)$ & $(3.453)$ & $(1.951)$
\end{tabular}

\footnotetext{
${ }^{6}$ La evidencia existente para España sugiere que el desempeño académico de los estudiantes universitarios está muy influido por los resultados en la educación preuniversitaria (véase, por ejemplo, Pérez \& Serrano, 2012).

${ }^{7}$ Esta estimación se ha realizado únicamente para los estudiantes que accedieron a la universidad a través de esta modalidad, que son la mayoría (más del 80\%) de los encuestados. Por una cuestión de espacio, en la tabla 5 aparecen únicamente los coeficientes de las variables de interés. 


$\begin{array}{llll}\text { Motivación } & 0.004 & -0.241 & 0.080 \\ \text { Vocación en el grado } & (0.007) & (0.172) & (0.097) \\ & 0.116 & 1.222 & 0.422 \\ \text { Repetidor } & (0.032)^{* * *} & (0.837) & (0.473) \\ & -0.309 & -1.944 & -1.609 \\ \text { Asistencia todas asignaturas: menos mitad } & (0.066) * * * & (1.727) & (0.976)^{*} \\ & 0.044 & -2.612 & 0.224 \\ \text { Asistencia todas asignaturas: más mitad } & (0.185) & (-4.757) & (2.688) \\ & 0.014 & -7.073 & -0.293 \\ \text { Asistencia todas asignaturas: casi siempre } & (0.173) & (4.441) & (2.509) \\ & 0.077 & -2.483 & 2.023 \\ \text { Horas de estudio a la semana } & (0.175) & (4.488) & (2.536) \\ & 0.006 & 0.073 & 0.205 \\ \text { Constante } & (0.004) & (0.096) & (0.054)^{* * *} \\ R^{2} & 4.714 & 26.120 & 5.116 \\ N & (0.338)^{* * *} & (8.875) * * * & (5.014) \\ & 0.27 & 0.23 & 0.32 \\ & 464 & 464 & 464\end{array}$

Nota: Se han introducido como variables de control también efectos fijos por clase.

Tabla 3. Influencia de trabajar habitualmente 30 horas o más en el rendimiento académico de los estudiantes

\begin{tabular}{|c|c|c|c|}
\hline & $\begin{array}{l}\text { Nota } \\
\text { Media } \\
\text { grado }\end{array}$ & $\begin{array}{l}\text { Créditos } \\
\text { aprobados } \\
\text { por año en } \\
\text { grado }\end{array}$ & $\begin{array}{l}\text { Créditos } \\
\text { aprobados en } \\
\text { semestre }\end{array}$ \\
\hline Trabajo habitual 30 o más horas & $\begin{array}{l}0.095 \\
(0.140)\end{array}$ & $\begin{array}{l}-5.180 \\
(3.618)\end{array}$ & $\begin{array}{l}-0.819 \\
(2.049)\end{array}$ \\
\hline Hombre & $\begin{array}{l}0.010 \\
(0.060)\end{array}$ & $\begin{array}{l}-5.386 \\
(1.570)^{* * *}\end{array}$ & $\begin{array}{l}-0.792 \\
(0.889)\end{array}$ \\
\hline Edad & $\begin{array}{l}0.022 \\
(0.009) * *\end{array}$ & $\begin{array}{l}0.914 \\
(0.236) * * *\end{array}$ & $\begin{array}{l}0.108 \\
(0.134)\end{array}$ \\
\hline Nacionalidad española & $\begin{array}{l}0.146 \\
(0.084)^{*}\end{array}$ & $\begin{array}{l}0.829 \\
(2.196)\end{array}$ & $\begin{array}{l}0.426 \\
(1.244)\end{array}$ \\
\hline Padre con estudios terciarios & $\begin{array}{l}-0.104 \\
(0.072)\end{array}$ & $\begin{array}{l}-0.649 \\
(1.899)\end{array}$ & $\begin{array}{l}-0.463 \\
(1.075)\end{array}$ \\
\hline Madre con estudios terciarios & $\begin{array}{l}0.074 \\
(0.073)\end{array}$ & $\begin{array}{l}0.334 \\
(1.901)\end{array}$ & $\begin{array}{l}1.143 \\
(1.077)\end{array}$ \\
\hline Renta del hogar media-baja & $\begin{array}{l}0.013 \\
(0.094)\end{array}$ & $\begin{array}{l}-0.620 \\
(2.459)\end{array}$ & $\begin{array}{l}-1.396 \\
(1.393)\end{array}$ \\
\hline Renta del hogar media-alta & $\begin{array}{l}-0.089 \\
(0.095)\end{array}$ & $\begin{array}{l}-0.409 \\
(2.491)\end{array}$ & $\begin{array}{l}-0.291 \\
(1.411)\end{array}$ \\
\hline Renta del hogar alta & $\begin{array}{l}0.027 \\
(0.131)\end{array}$ & $\begin{array}{l}1.405 \\
(3.445)\end{array}$ & $\begin{array}{l}2.924 \\
(1.951)\end{array}$ \\
\hline Motivación & $\begin{array}{l}0.001 \\
(0.007)\end{array}$ & $\begin{array}{l}-0.206 \\
(0.171)\end{array}$ & $\begin{array}{l}0.090 \\
(0.097)\end{array}$ \\
\hline Vocación en el grado & $\begin{array}{l}0.115 \\
(0.032) * * *\end{array}$ & $\begin{array}{l}1.191 \\
(0.835)\end{array}$ & $\begin{array}{l}0.421 \\
(0.473)\end{array}$ \\
\hline Repetidor & $\begin{array}{l}-0.315 \\
(0.066) * * *\end{array}$ & $\begin{array}{l}-2.007 \\
(1.720)\end{array}$ & $\begin{array}{l}-1.596 \\
(0.974)\end{array}$ \\
\hline $\begin{array}{l}\text { Asistencia todas asignaturas: menos } \\
\text { mitad }\end{array}$ & 0.067 & -3.259 & 0.062 \\
\hline & $(0.185)$ & $(4.756)$ & $(2.693)$ \\
\hline Asistencia todas asignaturas: más mitad & $\begin{array}{l}0.042 \\
(0.173)\end{array}$ & $\begin{array}{l}-7.707 \\
(4.434)\end{array}$ & $\begin{array}{l}-0.467 \\
(2.511)\end{array}$ \\
\hline Asistencia todas asignaturas: casi siem- & 0.118 & -3.305 & 1.788 \\
\hline
\end{tabular}




\begin{tabular}{llll} 
pre & & & \\
& $(0.175)$ & $(4.476)$ & $(2.535)$ \\
Horas de estudio a la semana & 0.006 & 0.067 & 0.203 \\
& $(0.004)^{*}$ & $(0.096)$ & $(0.054) * * *$ \\
Constante & 4.876 & 22.967 & 4.300 \\
& $(0.344) * * *$ & $(8.948) * *$ & $(5.067)$ \\
$R^{2}$ & 0.27 & 0.24 & 0.32 \\
$N$ & 464 & 464 & 464 \\
\hline
\end{tabular}

Nota: Se han introducido como variables de control también efectos fijos por clase.

Tabla 4. Influencia de trabajar habitualmente en el rendimiento académico de los estudiantes. Estimaciones alternativas con controles de motivación y capacidad

\begin{tabular}{|c|c|c|c|c|c|c|}
\hline & \multicolumn{3}{|c|}{ Trabajar habitualmente } & \multicolumn{3}{|c|}{ Trabajar habitualmente 30 horas o más } \\
\hline & $\begin{array}{l}\text { Nota } \\
\text { Media } \\
\text { grado }\end{array}$ & $\begin{array}{c}\text { Créditos } \\
\text { aprobados } \\
\text { por año en } \\
\text { grado }\end{array}$ & $\begin{array}{l}\text { Créditos } \\
\text { aprobados en } \\
\text { semestre }\end{array}$ & $\begin{array}{l}\text { Nota } \\
\text { Media } \\
\text { grado }\end{array}$ & $\begin{array}{l}\text { Créditos } \\
\text { aprobados } \\
\text { por año en } \\
\text { grado }\end{array}$ & $\begin{array}{l}\text { Créditos } \\
\text { aprobados en } \\
\text { semestre }\end{array}$ \\
\hline \multirow[t]{2}{*}{ Trabajo habitual } & -0.126 & -3.620 & -1.062 & 0.069 & -3.055 & 0.548 \\
\hline & $(0.083)$ & -2.576 & -1.537 & $(0.133)$ & -4.060 & -2.420 \\
\hline \multirow[t]{2}{*}{ Motivación } & -0.002 & -0.378 & -0.037 & -0.004 & -0.398 & -0.054 \\
\hline & $(0.006)$ & $(0.175)$ & $(0.105)$ & $(0.006)$ & $(0.175)$ & $(0.105)$ \\
\hline \multirow{2}{*}{$\begin{array}{l}\text { Vocación en el } \\
\text { grado }\end{array}$} & 0.070 & 1.250 & 0.675 & 0.069 & 1.135 & 0.671 \\
\hline & $(0.027)^{* *}$ & $(0.845)$ & $(0.504)$ & $(0.027)^{* *}$ & $(0.850)$ & $(0.506)$ \\
\hline \multirow{2}{*}{$\begin{array}{l}\text { Nota media } \\
\text { bachillerato }\end{array}$} & 0.219 & 5.207 & 3.255 & 0.219 & 5.098 & 3.256 \\
\hline & $(0.033)^{* * *}$ & $(1.028) * * *$ & $(0.614) * * *$ & $(0.033)^{* * *}$ & $(1.034)^{* * *}$ & $(0.616)^{* * *}$ \\
\hline \multirow{2}{*}{$\begin{array}{l}\text { Nota media } \\
\text { PAU }\end{array}$} & 0.025 & 0.851 & 0.458 & 0.026 & 0.816 & 0.462 \\
\hline & $(0.018)$ & $(0.574)$ & $(0.342)$ & $(0.019)$ & $(0.576)$ & $(0.343)$ \\
\hline$R^{2}$ & 0.46 & 0.37 & 0.39 & 0.46 & 0.37 & 0.39 \\
\hline$N$ & 383 & 383 & 383 & 383 & 383 & 383 \\
\hline
\end{tabular}

En segundo lugar se ha estimado mediante variables instrumentales. Tal y como se ha señalado, una de las principales dificultades de esta técnica estriba en encontrar un conjunto de instrumentos apropiado, el cual debe cumplir dos supuestos (véase, por ejemplo, Greene, 2003): el de relevancia (los instrumentos deben estar suficientemente relacionados con la variable endógena a la que sustituye en las estimaciones) y el de exogeneidad (los instrumentos deben influir en la variable dependiente únicamente a través de su efecto sobre la variable endógena, de tal forma que no deben estar correlacionados con el término de error). En estudios previos sobre el mismo tema de investigación los instrumentos elegidos habitualmente 
han sido características que miden el nivel socioeconómico de las familias, en tanto se entiende que el mismo pudiera estar relacionado con la elección de trabajar (asumiendo que los estudiantes que forman parte de las familias con menos recursos necesitan trabajar en mayor medida con el fin de cubrir los costes asociados con sus estudios universitarios) pero no con el rendimiento académico de los estudiantes una vez alcanzada una determinada etapa educativa (Ruesga et al., 2014). Nuestra estimación mediante variables instrumentales considera las mismas variables de control que las de las estimaciones mediante mínimos cuadrados ordinarios, con la excepción de las medidas de motivación y capacidad, y, siguiendo a Ruesga et al. (2014) se han utilizado dos instrumentos: una medida del estatus socioeconómico de las familias (en nuestro caso, una medida directa correspondiente a la categoría relativa de la renta del hogar en el conjunto del país) y el turno de estudios (con una variable dicotómica que diferencia entre turno matutino o vespertino).

Tabla 5. Influencia de trabajar habitualmente en el rendimiento académico de los estudiantes. Estimaciones alternativas mediante variables instrumentales

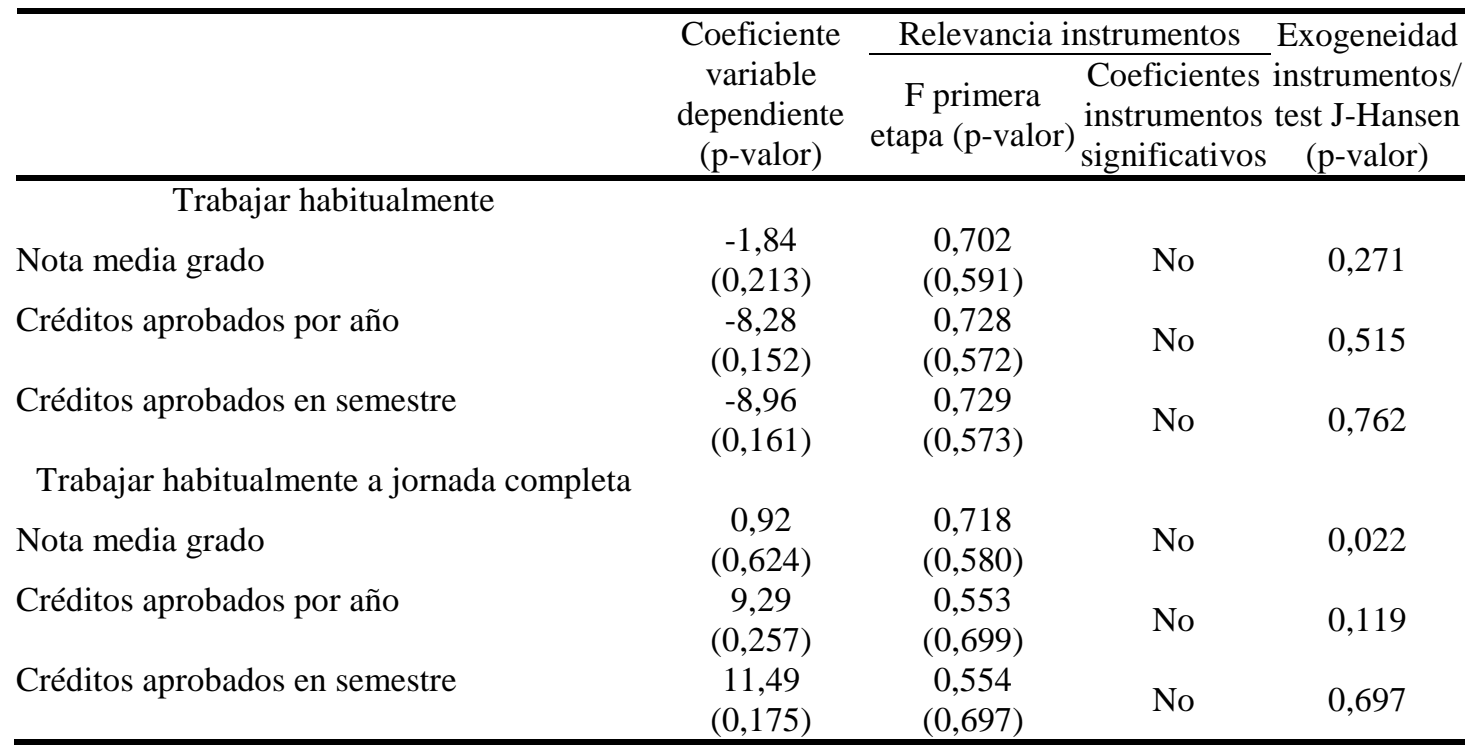

Los resultados de las estimaciones mediante variables instrumentales aparecen en la tabla 5 de forma separada para cada modelo considerado. Si bien en ningún caso el coeficiente estimado para la variable dependiente (alternativamente, trabajar habitualmente o hacerlo a jornada completa) es estadísticamente significativo a los niveles convencionales, los mismos muestran que aunque en general puede aceptarse el supuesto de exogeneidad de los instrumentos (los p-valores del test de J-Hansen son en prácticamente todos los casos elevados y 
superiores a los niveles convencionales de significatividad), en ninguno de los modelos estimados los instrumentos cumplen el criterio de relevancia. Así, en todos los casos los resultados de la primera etapa de la estimación de variables instrumentales (donde se estima un modelo en el que la variable potencialmente endógena se toma como variable dependiente y los instrumentos como variables independientes) muestran que los coeficientes estimados para los distintos instrumentos no son significativos y que los valores del estadístico $\mathrm{F}$ que permite contrastar la significatividad conjunta de todos ellos son relativamente pequeños (y, en consecuencia, sus p-valores relativamente grandes). Esta evidencia indica, en consecuencia, que, al menos en el caso de la población analizada, los instrumentos utilizados son débiles e irrelevantes y su uso no es apropiado, pues la estimación mediante variables instrumentales estaría sesgada lo que, entre otras cuestiones, impediría el uso de los métodos tradicionales de inferencia (Cameron \& Trivedi, 2005) $)^{8}$.

\section{Conclusiones}

La finalidad de este artículo es examinar la incidencia y las características de simultanear trabajo y estudios en el alumnado universitario español, así como examinar sus posibles efectos sobre el rendimiento académico. Entendemos que este análisis puede ayudar a disponer de un diagnóstico preciso de las dificultades potenciales que encuentran los estudiantes que también trabajan (lo que podría permitir, entre otras circunstancias, diseñar tratamientos específicos en el proceso de aprendizaje para este tipo de estudiantes en caso de que fuera necesario) y que es especialmente relevante en el caso español, para el que la evidencia previa sobre estas cuestiones es muy escasa.

El instrumento básico para el desarrollo de la investigación es una encuesta suministrada a estudiantes de la Universidad de Alicante. Se trata de una encuesta con una muy elevada tasa de respuesta y con información muy completa tanto sobre las características individuales y socioeconómicas de los estudiantes y sus familias (incluyendo el cuartil que ocupa la familia en la distribución de la renta española) como sobre los potenciales determinantes del

\footnotetext{
${ }^{8}$ Estas estimaciones se han replicado tanto utilizando posibles instrumentos alternativos, como el nivel de estudios de los padres y la modalidad en que se cursa el grado (completo o parcial), como estimando alternativamente mediante variables instrumentales usando el estimador LIML (limited information maximum likelihood), el cual es más apropiado que el estimador estándar en dos etapas (2SLS) cuando la muestra es pequeña y los instrumentos son débiles, como es el caso (véanse, por ejemplo, Anderson, Kunitomo \& Sawa, 1982; y Kunitomo 
rendimiento académico (incluyendo la motivación, la vocación y el esfuerzo relacionados con los estudios) así como si se desempeña una actividad laboral y las características de ésta. Un aspecto destacable de la misma es que la información suministrada por los estudiantes en la encuesta se complementa con información adicional proveniente de los registros universitarios, una circunstancia que permite enriquecer notablemente el análisis, ya que permite disponer de más información y reducir significativamente los errores de medida en algunas de las variables de mayor relevancia.

La evidencia obtenida permite realizar, en primer lugar, una detallada caracterización de los estudiantes universitarios que trabajan en España. Así, se observa que en torno a una tercera parte de los estudiantes universitarios simultanea los estudios universitarios con un trabajo remunerado, lo que confirma que se trata de un fenómeno relativamente significativo en nuestro país, así como que en torno a la mitad de los estudiantes que trabajan lo hacen de forma habitual. Se constata también que los estudiantes que trabajan habitualmente perciben generalmente un impacto negativo de trabajar en su desempeño académico, así como que una proporción no desdeñable de los mismos trabaja mayoritariamente en trabajos no relacionados con el contenido de sus estudios y en situación legal, lo hace por necesidad y no podría estudiar sin los ingresos asociados a su trabajo. Los resultados de la estimación de modelos multivariantes muestran, por su parte, que ciertas características están asociadas de forma significativa a una mayor incidencia de trabajar, en tanto que la probabilidad de trabajar es mayor entre los estudiantes de mayor edad, los no nacionales y los que presentan una mayor motivación.

Por otra parte, los resultados obtenidos a partir de la estimación de técnicas de análisis multivariante de modelos en los que se considera diversas medidas del logro académico y se controla por un amplio conjunto de factores (incluyendo características individuales y familiares, medidas de motivación general y de esfuerzo académico) sugieren que, en contraste con la propia percepción manifestada por los estudiantes, trabajar regularmente no tiene aparentemente un impacto significativo en sus resultados académicos. Este resultado concuerda con los hallazgos de estudios previos para otros países en el sentido de que trabajar no es perjudicial para los logros académicos de los estudiantes universitarios, pero contrasta con la evidencia obtenida por Ruesga et al. (2014) para el caso español. Aunque esta discrepancia en el 
sentido de los resultados podría deberse a las distintas fuentes de información, métodos analíticos y poblaciones objeto de investigación de ambos estudios, la misma concuerda en cualquier caso con el carácter no concluyente de la evidencia internacional previa sobre los efectos de trabajar en el rendimiento académico de los estudiantes universitarios y es indicativa de la necesidad de profundizar en la investigación de esta cuestión para nuestro país.

\section{Agradecimientos}

El presente trabajo se enmarca en el seno del Programa de Redes de investigación en docencia universitaria del Vicerrectorado de Calidad e Innovación Educativa-Instituto de Ciencias de la Educación de la Universidad de Alicante (convocatoria 2015-16), ref.: 3478. Además, los autores desean hacer constar su agradecimiento a la financiación recibida del Ministerio de Economía y Competitividad en el marco del Plan Estatal de I+D+i, ref. CSO2014-55780-C32-P

\section{Referencias}

Alonso, J. (1992). Motivar en la adolescencia: Teoría, evaluación e intervención. Madrid: Servicio de Publicaciones de la Universidad Autónoma de Madrid.

Anderson, T. W.; Kunitomo, N. y Sawa, T. (1982). Evaluation of the distribution function of the limited information maximum likelihood estimator. Econometrica: Journal of the Econometric Society, 1009-1027. [DOI: 10.2307/1912774]

Angrist, J.D. y Pischke, J.S. (2009). Mostly harmless econometrics: An empiricist's companion. Princeton: Princeton University Press.

Baum, C. F. (2006). An introduction to modern econometrics using Stata. College Station: Stata Press.

Beffy, M., D. Fougère y A. Maurel (2009). L’impact du travail salarié des étudiants sur la réussite et la poursuite des études universitaires. Économie et Statistique, 422. [DOI: $10.3406 / 2009.8017]$

Cameron, A.C. y Trivedi, P. K. (2005). Microeconometrics: methods and applications. Cambridge: Cambridge University Press.

Canabal, M. E. (1998). College student degree of participation in the labor force: Determinants and relationship to school performance. College Student Journal, 32(4), 597605. [DOI: 10.3102/00346543076001063] 
Castejón, J.L. (2014). Aprendizaje y rendimiento académico. Alicante: Editorial Club Universitario.

Curtis, S. (2007). Students' perceptions of the effects of term-time paid employment. Education+Training, 49(5), 380-390. [DOI: 10.1108/00400910710762940]

Dundes, L. \& Marx, J. (2006). Balancing Work and Academics in College: Why Do Students Working 10 to 19 Hours per Week Excel? Journal of College Student Retention: Research, Theory and Practice, 8 (1) [DOI: 10.2190/7UCU-8F9M-94QG-5WWQ]

Furr, S. R., y Elling, T. W. (2000). The influence of work on college student development. NASPA Journal, 37(2), 454-470. [DOI: 10.2202/1949-6605.1108]

Gleason, P. M. (1993). College student employment, academic progress, and post-college labor market success. Journal of Student Financial Aid, 23(2), 5-14.

Gordon, R. A. (1968). Issues in multiple regression. American Journal of Sociology, 73, 592616. [DOI: $10.1086 / 224533]$

Greene, W. H. (2003). Econometric analysis. Pearson Education India.

Hammes, J. F. y Haller, E. J. (1983). Making ends meet: Some of the consequences of parttime work for college students. Journal of College Student Personnel, 24(6), 529-535.

Hawkins, C.A.; Smith, M.L., Hawkins, R.C. y Grant, D. (2005). The relationship among hours employed, perceived work interference, and grades as reported by undergraduate social work students. Journal of Social Work Education, 41(1), 13-27. [DOI: 10.5175/JSWE.2005.200202122]

Hood, A, A. Craig y B. Ferguson (1992). The impact of athletics, part-time employment and other academic activities on academic achievement. Journal of College Student Development, 33, 447-453.

Kunitomo, N. y Matsushita, Y. (2008). Improving the rank-adjusted Anderson-Rubin test with many instruments and persistent heteroscedasticity, No. CIRJE-F-588, CIRJE.

Lammers, W. J., Onwuegbizie, A. J., y Slate, J. R. (2001). Academic success as a function of the gender, class, age, study habits, and employment of college students. Research in the Schools, 8(2), 71-81.

Lang, K. B. (2012). The similarities and differences between working and non-working students at a mid-sized American public university. College Student Journal, 46(2), 243.

Lillydahl, J. (1990). Academic achievement and part-time employment of High school students. Journal of Economic Education, 21 (3), 307-316. [DOI: 10.1080/00220485.1990.10844678] 
Lyons, T. F., Krachenberg, A. R. y Henke, J. W. (1986). Academic performance and work: A methodological critique and review. Social and Behavioral Sciences Documents, $16(1), 34$.

Montero, I. y Alonso, J. (1992). El cuestionario MAPE-II. En J. Alonso (Ed.), Motivar en la adolescencia: Teoría, evaluación e intervención (pp. 205-231). Madrid: Servicio de Publicaciones de la Universidad Autónoma de Madrid.

Nonis, S. A. y Hudson, G. I. (2006). Academic performance of college students: Influence of time spent studying and working. Journal of Education for Business, 81(3), 151-159. [DOI: 10.3200/JOEB.81.3.151-159]

Pascarella, E. T., Bohr, L., Amaury, N., Desler, M. y Zusman, B. (1994). Impacts of oncampus and off-campus work on first year cognitive outcomes. Journal of College Student Development, 35, 364-370.

Pascarella, E. T., Edison, M. T., Nora, A., Hagedorn, L. S. y Terenzini, P. T. (1998). Does work inhibit cognitive development during college? Educational Evaluation and Policy Analysis, 20(2), 75-93. [DOI: 10.3102/01623737020002075]

Paul, H. (1982). The impact of outside employment on student academic achievement in Macroeconomic principles. Journal of Economic Education, 13, 51-56. [DOI: 10.1080/00220485.1982.10844995]

Pelechano, V. (1975). Cuestionario MAE. Madrid: Fraser Española División de Psicología.

Pérez García, F.; Serrano, L. (2012). Universidad, Universitarios y Productividad en España, Fundación BBVA.

Pike, G. R., Kuh, G. D. y Massa-McKinley, R. C. (2008). First-year students' employment, engagement, and academic achievement: Untangling the relationship between work and grades. Journal of Student Affairs Research and Practice, 45(4), 1012-1034. [DOI: 10.2202/1949-6605.2011]

Richardson, J. J., Kemp, S., Malinen, S. y Haultain, S. A. (2013). The academic achievement of students in a New Zealand university: Does it pay to work? Journal of Further and Higher Education, 37(6), 864-882. [DOI: 10.1080/0309877X.2012.699517]

Riggert, S. C., Boyle, M., Petrosko, J. M., Ash, D. y Rude-Parkins, C. (2006). Student employment and higher education: Empiricism and contradiction. Review of Educational Research, 76(1), 63-92). [DOI: 10.3102/00346543076001063] 
Ruesga, S.M.; da Silva, J. y Monsueto, S.E. (2014). Estudiantes universitarios, experiencia laboral y desempeño académico en España. Revista de Educación, 345. Julioseptiembre, pp. 67-95. [DOI: 10.4438/1988-592X-RE-2014-365-265]

Quintini, G. (2015). Working and learning: A diversity of patterns. OECD Social, Employment and Migration Working Papers, No. 169.

Stern, D. y Nakata, F. (1991). Paid employment among US college students: Trends, effects, and possible causes. The Journal of Higher Education, 25-43. [DOI: 10.1080/00221546.1991.11774104]

Stern, D. Finkelstein, N; Urquiola, M. y Cagampang, H. (1997). What difference does it make if school and work are connected? Evidence on Co-operative education in the United States. Economics of Education Review, 16 (3), 213-229. [DOI: 10.1016/S0272-7757(96)00074-X]

Stinebrickner, T. y Stinebrickner, R. (2003). Working during school and academic performance. Journal of Labor Economics, 21 (2), 473-491. [DOI: 10.1086/345565]

Tejedor, F. J. y García-Valcarcel, A. (2007). Causas del bajo rendimiento del estudiante universitario (en opinión de los profesores y alumnos). Propuestas de mejora en el marco del EEES. Revista de Educación, 342, enero-abril, 443-473. [DOI: 10.4438/1988592X-RE-2007-263-157]

Warren, J.R. (2002). Reconsidering the relationship between student employment and academic outcomes: A new theory and better data. Youth\&Society, 33(3), 366-393. [DOI: 10.1177/0044118X02033003002]

Wenz, M. y Yu, W.C. (2010). Term-time employment and the academic performance of undergraduates. Journal of Education Finance, 35(4), 358-373. [DOI:
[DO 10.1353/jef.0.0023] 


\section{Anexo}

Tabla A.1. Descriptivos de las variables

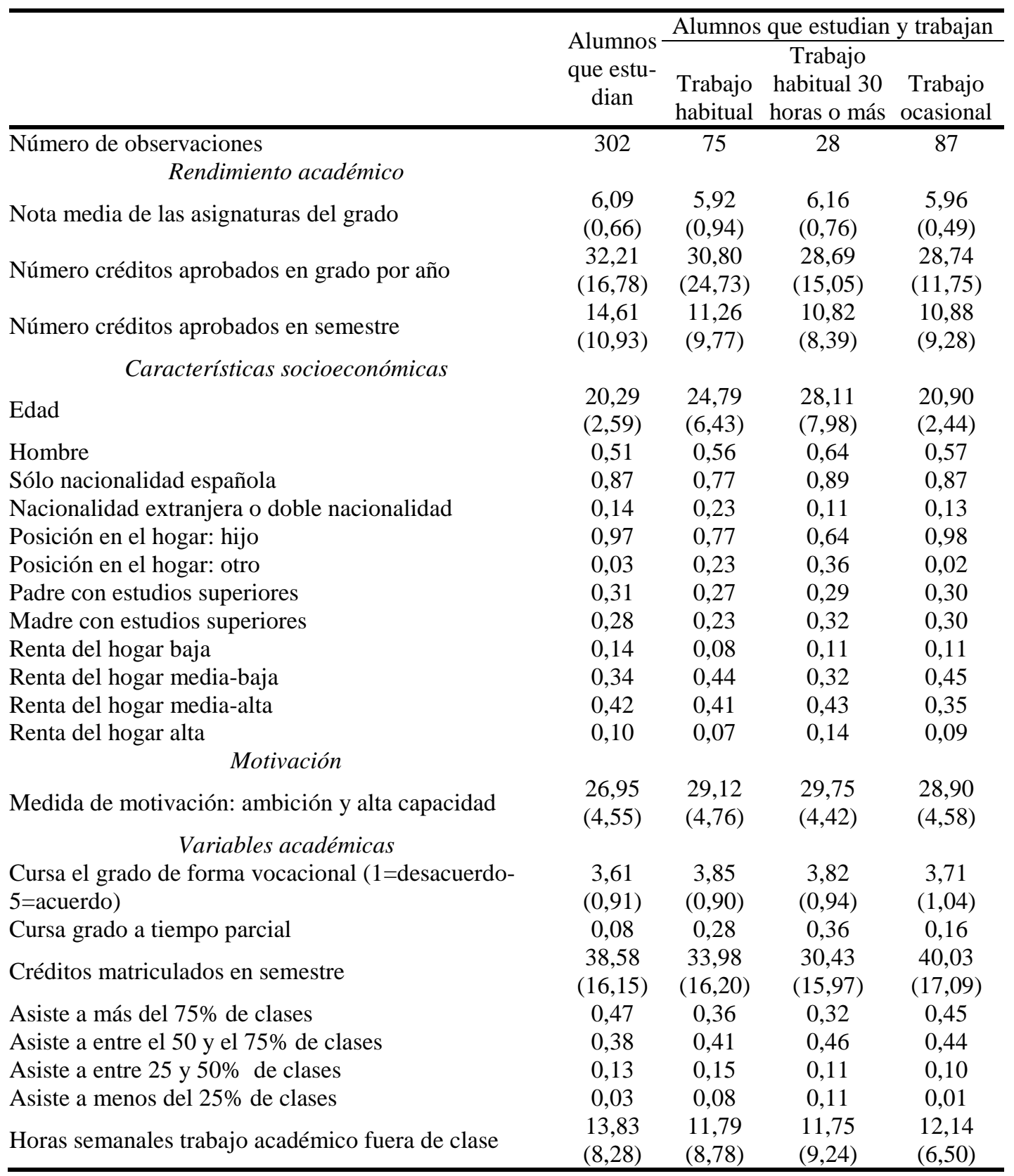

Nota: Entre paréntesis aparece la desviación estándar de la variable. 
Tabla A.2. Características de los estudiantes que trabajan y de sus empleos

\begin{tabular}{|c|c|c|c|}
\hline & \multicolumn{2}{|c|}{ Trabajo habitual } & \multirow{2}{*}{$\begin{array}{l}\text { Trabajo } \\
\text { ocasional }\end{array}$} \\
\hline & Total & $\begin{array}{l}30 \text { horas } \\
\text { o más }\end{array}$ & \\
\hline Horas de trabajo semanales en trabajo habitual & $\begin{array}{c}21,87 \\
(12,47)\end{array}$ & $\begin{array}{l}36,46 \\
(5,38)\end{array}$ & - \\
\hline Situación: sólo estudio & 0,04 & 0,04 & 0,29 \\
\hline Situación: principalmente estudio & 0,31 & 0,18 & 0,64 \\
\hline Situación: principalmente trabajo & 0,33 & 0,46 & 0,03 \\
\hline Situación: trabajo y estudio & 0,32 & 0,32 & 0,03 \\
\hline Ocupación del empleo cualificada & 0,17 & 0,24 & 0,10 \\
\hline Ocupación del empleo semicualificada & 0,73 & 0,68 & 0,70 \\
\hline Ocupación del empleo no cualificada & 0,10 & 0,08 & 0,21 \\
\hline Sector del empleo: primario & 0,00 & 0,04 & 0,06 \\
\hline Sector del empleo: industria & 0,01 & 0,00 & 0,04 \\
\hline Sector del empleo: construcción & 0,03 & 0,00 & 0,01 \\
\hline Sector del empleo: servicios & 0,96 & 0,96 & 0,88 \\
\hline $\begin{array}{l}\text { Motivo de trabajar: necesidad (ingresos básicos para economía } \\
\text { familiar) }\end{array}$ & 0,36 & 0,57 & 0,24 \\
\hline Motivo de trabajar: ayudar a la economía familiar & 0,75 & 0,79 & 0,77 \\
\hline Trabajo habitualmente en situación legal (1-5) & $\begin{array}{c}2,44 \\
(1,45)\end{array}$ & $\begin{array}{c}2,36 \\
(1,52)\end{array}$ & $\begin{array}{c}2,88 \\
(1,39)\end{array}$ \\
\hline Podría cursar estudios sin ingresos del empleo (1-5) & $\begin{array}{c}2,81 \\
(1,54)\end{array}$ & $\begin{array}{c}2,46 \\
(1,55)\end{array}$ & $\begin{array}{c}3,80 \\
(1,40)\end{array}$ \\
\hline Empleo no relacionado con el contenido del grado (1-5) & $\begin{array}{c}2,74 \\
(1,60)\end{array}$ & $\begin{array}{c}2,68 \\
(1,72)\end{array}$ & $\begin{array}{c}2,11 \\
(1,36)\end{array}$ \\
\hline $\begin{array}{l}\text { Empleo afecta negativamente al tiempo para preparación académica } \\
(1-5)\end{array}$ & $\begin{array}{c}3,73 \\
(0,90)\end{array}$ & $\begin{array}{c}3,82 \\
(1,02)\end{array}$ & $\begin{array}{c}2,68 \\
(1,29)\end{array}$ \\
\hline Rendimiento académico mejoraría si no trabajara (1-5) & $\begin{array}{c}4,18 \\
(0,88)\end{array}$ & $\begin{array}{c}4,50 \\
(0,69)\end{array}$ & $\begin{array}{c}2,87 \\
(1,43)\end{array}$ \\
\hline
\end{tabular}

Nota: Entre paréntesis aparece la desviación estándar de la variable. 